\title{
RELIABLE ROBUST CONTROLLER FOR HALF-CAR ACTIVE SUSPENSION SYSTEMS BASED ON HUMAN-BODY DYNAMICS
}

\author{
UDC 681.5:629.3
}

\author{
Mohammad Gudarzi \\ Young Researchers and Elites Club, Damavand Branch, Islamic Azad University, Iran
}

\begin{abstract}
The paper investigates a non-fragile robust control strategy for a half-car active suspension system considering human-body dynamics. A 4-DoF uncertain vibration model of the driver's body is combined with the car's model in order to make the controller design procedure more accurate. The desired controller is obtained by solving a linear matrix inequality formulation. Then the performance of the active suspension system with the designed controller is compared to the passive one in both frequency and time domain simulations. Finally, the effect of the controller gain variations on the closed-loop system performance is investigated numerically.
\end{abstract}

Key Words: Body Acceleration, Dynamic Modeling, LMIs, Driver's Biodynamics

\section{INTRODUCTION}

Passive, semi-active and active suspensions are three main types of car suspensions that are used by automotive industries. All suspension systems aim to improve the car performance consisting of ride comfort, handling, road holding, suspension deflection, static deflection, etc. Conventional passive suspensions are effective only in a certain frequency range and optimal design performance cannot be achieved when the system and its operating conditions are changed. On the contrary, it has been well recognized that active suspensions have a great potential to meet the tight performance requirements demanded by users. Therefore, in recent years more and more attention has been devoted to the development of active suspensions and various approaches have been proposed to solve the crucial problem of designing a suitable control law for these active suspension systems [1-3].

Most of the present studies on active suspensions are concerned with vibration reduction of the sprung mass containing vertical acceleration of Center of Gravity $(\mathrm{CoG})$,

Received May 16, 2016 / Accepted June 27, 2016

Corresponding author: Mohammad Gudarzi

Young Researchers and Elites Club, Damavand Branch, Islamic Azad University, Iran

E-mail: mohammad.gudarzi@gmail.com 
pitch acceleration and roll acceleration [4-8]. However, passengers do not sit on the CoG of the vehicle and so, vertical, roll and pitch motion of the vehicle affect the acceleration of the passenger's seats. In addition, around the resonance frequency of the seated human, the passenger's vibration magnitude due to the seated human dynamics becomes larger than the seated position's vibration of the sprung mass. Moreover, these vibrations cause the driver's whole body to vibrate. Undesirable effects of the driver's body vibration are experienced when the exposure time is longer than the recommended standard set by ISO 2631-1 [9]. So, to take into consideration the driver's biodynamics in the controller design can improve its performance [10].

In addition to aforementioned aspects, another important goal of the controller design for active suspension systems is to maintain the robustness of the closed-loop systems [11]. In real cars, the total vehicle mass varies due to the changes in passenger load and cargo while characteristics of the actuators change due to aging and nonlinearities. When parameters in the plants change like this, the control performance specified in the design stage tends to deteriorate especially in LQG controllers [12]. However, a constant performance is desired in automotive suspensions, so, this deterioration should be kept as small as possible. Therefore, the analysis and synthesis of robust control for active suspension systems have become a research concern in recent years [13]. The application of the standard robust control theory has an assumption that the controller can be realized exactly. However, in practice, many physical limitations such as the effects of finite word length in any digital system, round-off errors in numerical arithmetics, inherent imprecision in analog devices, etc. lead to a loss of precision in controller implementation. Accordingly, even though the designed controllers are robust with respect to system uncertainties, they may be very sensitive to their own uncertainties. Recently, much attention has been paid to the so-called fragility problems of controllers [14-15]. This problem is basically associated with performance decrease of a closed-loop system due to the inaccuracies in the controller implementation [16]. In recent years, many works have been made to solve the non-fragile controller design problem for linear systems [17-18]. Some state feedback non-fragile $\mathrm{H} \infty$ controller design method with respect to additive and multiplicative norm-bounded controller gain variations are given in some literature [18-19]. In addition, this kind of controller has been implemented on an active suspension system [20].

The above review indicates that while there exists a notable body of literature on the active car suspension control, a comprehensive investigation on active suspension systems considering accurate human biodynamics based on robust non-fragile controller design seem to be absent. In this study, the problem of robust non-fragile $\mathrm{H} \infty$ controller design for a half-car suspension system with norm-bounded parameter uncertainties and controller gain variations is investigated. In order to obtain a better insight of the suspension system performance, a 4-DoF uncertain vibration model of the driver's body is combined with the car's model. Due to no explicit inverse of mass matrix existing in controller design approach, the uncertainties in mass, damping and stiffness can be described more naturally and directly. Considered additive controller gain variations in this approach, also, makes this approach more robust and more applicable in engineering practice. Finally, the desired controller has been obtained by solving a set of linear matrix inequalities (LMIs) using MATLAB; then the frequency and time domain responses of the active suspension are compared to the passive ones and the effect of controller gain variations on the closed-loop system performance is investigated. 


\section{DYNAMIC MODELING}

A half-vehicle model, which is equipped with an active suspension between each unsprung mass and the sprung mass, is shown in Fig. 1. The detailed equations of motion for this half-car model, which are bounce, pitch and each unsprung motions are as given in [21]. The schematic model of the driver's biodynamic consisting of the lumped human linear seat model is illustrated in Fig. 2. Considered model for the driver is based on a 4DOF human body presented in [21]; the related equations could be found there as well. By assuming that the passenger is sitting in the front seat, the obtained dynamic equations by adding structured uncertainties can be rearranged as:

$$
\left(M+\Delta_{M}\right) \ddot{z}(t)+\left(C+\Delta_{C}\right) \dot{z}(t)+\left(K+\Delta_{K}\right) z(t)=B u(t)+B_{w} w(t),
$$

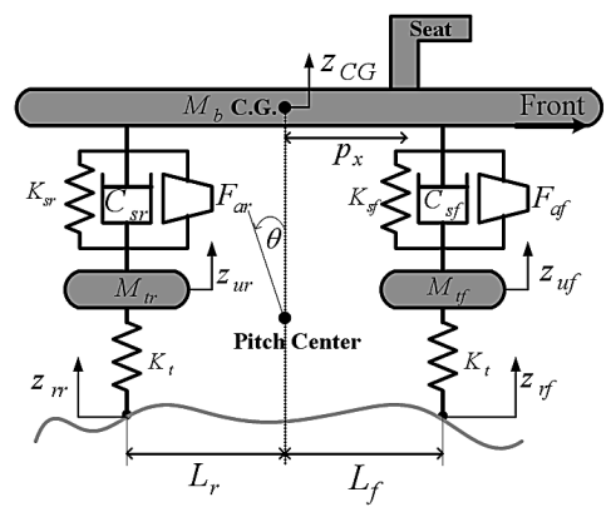

Fig. 1 Half-vehicle model

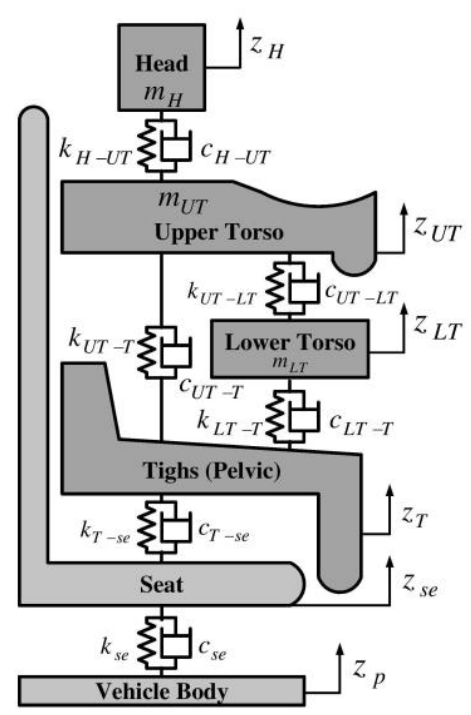

Fig. 2 Passenger model 
where $z(t)=\left[z_{u f}, z_{u r}, z_{C G}, \theta, z_{H}, z_{U T}, z_{L T}, z_{T}, z_{s e}\right]^{T} \in R^{9}$ is the displacement and rotation vector, $u(t)=\left[F_{a f}, F_{a r}\right]^{T}$ is the control input vector, and $w(t)=\left[z_{r f}, z_{r r}\right]^{T}$ is the external disturbance from the road. $M \in R^{9 \times 9}, C \in R^{9 \times 9}$ and $K \in R^{9 \times 9}$ are the mass, damping, and stiffness matrices, respectively; $\Delta_{M}, \Delta_{C}$ and $\Delta_{K}$ are corresponding perturbations, $M \in R^{9 \times 2}$ is the input matrix and $B_{w} \in R^{9 \times 2}$ is the disturbance matrix.

By considering state $q(t)=\left[z^{T}(t), \dot{z}^{T}(t)\right]^{T}$, Eq. (6) can be written as:

$$
\left[\begin{array}{cc}
I & 0 \\
0 & M+\Delta_{M}
\end{array}\right] \dot{q}(t)=\left[\begin{array}{cc}
0 & I \\
-K-\Delta_{K} & -C-\Delta_{C}
\end{array}\right] q(t)+\left[\begin{array}{l}
0 \\
B
\end{array}\right] u(t)+\left[\begin{array}{c}
0 \\
B_{w}
\end{array}\right] w(t) .
$$

Here, $w(t)$ is assumed to be an energy-bounded signal (i.e., $w(t) \in L_{2}[0, \infty)$ ). The objective or measurement signal $z_{o}(t)$ to be controlled is considered as

$$
z_{\mathrm{o}}(t)=C_{d} z(t)+C_{v} \dot{z}(t)
$$

where $C_{d} \in R^{1 \times 9}$ and $C_{v} \in R^{1 \times 9}$. The system can be formulated as:

$$
\begin{aligned}
& \left(\mathcal{E}+\Delta_{\mathcal{E}}\right) \dot{q}(t)=\left(\mathcal{A}+\Delta_{\mathcal{A}}\right) q(t)+\mathcal{B} u(t)+\mathcal{B}_{w} w(t), \\
& z_{\mathrm{o}}(t)=\mathcal{L} q(t),
\end{aligned}
$$

where:

$$
\begin{aligned}
& \mathcal{E}=\left[\begin{array}{cc}
I & 0 \\
0 & M
\end{array}\right], \Delta_{\mathcal{E}}=\left[\begin{array}{cc}
0 & 0 \\
0 & \Delta_{M}
\end{array}\right], \mathcal{A}=\left[\begin{array}{cc}
0 & I \\
-K & -C
\end{array}\right], \Delta_{\mathcal{A}}=\left[\begin{array}{cc}
0 & 0 \\
-\Delta_{K} & -\Delta_{C}
\end{array}\right], \\
& \mathcal{B}=\left[\begin{array}{l}
0 \\
B
\end{array}\right], \mathcal{B}=\left[\begin{array}{l}
0 \\
B
\end{array}\right], \mathcal{B}_{w}=\left[\begin{array}{c}
0 \\
B_{w}
\end{array}\right], \mathcal{L}=\left[\begin{array}{ll}
C_{d} & C_{v}
\end{array}\right]
\end{aligned}
$$

Uncertainty $\Delta_{M}$ is assumed to satisfy:

$$
\Delta_{M} M^{-1} \leq \delta<1
$$

where $\|$.$\| refers to the Euclidean norm of a vector or matrix. Equation (5) implies that$ $\left\|\Delta_{E} E^{-1}\right\| \leq \delta<1$. Note that the condition (5) ensures that $E+\Delta_{E}$ is non-singular. In addition:

$$
\begin{aligned}
& \Delta_{K}=L_{k} F_{k} E_{k}, \\
& \Delta_{C}=L_{c} F_{c} E_{c},
\end{aligned}
$$

where $F_{k} \leq 1, F_{c} \leq 1, L_{k}, L_{c}, E_{k}, E_{c}$ are known constant matrices which characterize the influence of uncertain parameters $F_{c}, F_{k}$ in nominal damping matrix $C$ and stiffness matrix $K$, respectively. The uncertainties in active suspension system (1) satisfying Eqs. (5) and (6) are said to be admissible. Therefore:

$$
\Delta_{\mathcal{A}}=-\left[\begin{array}{c}
0 \\
L_{k}
\end{array}\right] F_{k}\left[\begin{array}{ll}
E_{k} & 0
\end{array}\right]-\left[\begin{array}{c}
0 \\
L_{c}
\end{array}\right] F_{c}\left[\begin{array}{ll}
0 & E_{c}
\end{array}\right]=\mathfrak{L}_{k} F_{k} \mathcal{E}_{k}+\mathfrak{L}_{c} F_{c} \mathcal{E}_{c}
$$

with:

$$
\mathfrak{L}_{k}=-\left[\begin{array}{c}
0 \\
L_{k}
\end{array}\right], \mathcal{E}_{k}=\left[\begin{array}{ll}
E_{k} & 0
\end{array}\right], \mathfrak{L}_{c}=-\left[\begin{array}{c}
0 \\
L_{c}
\end{array}\right], \quad \mathcal{E}_{c}=\left[\begin{array}{ll}
0 & E_{c}
\end{array}\right]
$$



by:

The control force, utilizing both displacement and velocity feedback signals, is obtained

$$
u(t)=\left(F_{d}+\Delta_{f d}\right) z(t)+\left(F_{v}+\Delta_{f v}\right) \dot{z}(t),
$$

where $F_{d} \in R^{2 \times 9}, F_{v} \in R^{2 \times 9}$ are the feedback gain vectors for the displacement and the velocity, respectively, and $\Delta_{f d}, \Delta_{f v}$ their corresponding uncertainties. This can be rearranged as:

$$
u(t)=\left(\mathcal{F}+\Delta_{\mathcal{F}}\right) q(t)
$$

where $F \in R^{2 \times 18}$ is the state feedback gain to be calculated, $F=\left[F_{d}, F_{v}\right], \Delta_{F}=\left[\Delta_{f d} \Delta_{f v}\right]$ is a norm-bounded feedback gain variation in the form of [19]:

$$
\Delta_{\mathcal{F}}=\chi_{f} F_{f} \mathcal{E}_{f},
$$

where $\chi_{f}, E_{f}$ are describing the uncertainty structure and they are known constant matrices of appropriate dimensions, and for the matrix $F_{f}$, we have $F_{f} \leq 1$.

Ride comfort quantified by driver's head vertical acceleration, suspension deflection limitation, road holding and actuator saturation are considered as controlled values.

$$
\begin{aligned}
& z_{o 2}(t)=\left[\begin{array}{ll}
z_{u f}(t) & z_{u r}(t)
\end{array}\right]^{T} \leq\left[\begin{array}{ll}
z_{\max f} & z_{\max r}
\end{array}\right]^{T} \\
& z_{o 1}(t)=\ddot{z}_{H}(t) \\
& z_{o 3}(t)=\left[\left|\frac{K_{f} z_{t f}(t)}{\bar{f}_{s f}} \| \frac{K_{r} z_{t r}(t)}{\bar{f}_{s r}}\right|\right] \leq\left[\begin{array}{ll}
1 & 1
\end{array}\right]^{T} \\
& z_{o 4}(t)=\left[\begin{array}{ll}
F_{a f}(t) & F_{a r}(t)
\end{array}\right]^{T} \leq\left[\begin{array}{ll}
F_{\max f} & F_{\max r}
\end{array}\right]^{T}
\end{aligned}
$$

The control design objective is to minimize $H_{\infty}$ norm of the closed-loop transfer function from output disturbances $w(t)$ to measurement signal $z_{o}(t), T_{z w}(s)_{\infty}$, so that it remains below a specified bounded value $\gamma>0$ for all admissible plant uncertainties and gain variations in $F$ (control). Such a designed control law is known as a non-fragile $H_{\infty}$ state feedback controller.

\section{CONTROLLER DESIGN}

In this section, a solution for the problem of robust non-fragile $H_{\infty}$ state feedback control for the active seat suspension system (4) in which both robust closed-loop stability and robust $\mathrm{H} \infty$ performance are achieved in spite of parametric uncertainties and controller gain variations is considered. System (4) with the state feedback control gain in Eq. (9) becomes:

$$
\begin{gathered}
\left(\mathcal{E}+\Delta_{\mathcal{E}}\right) \dot{q}(t)=\left(\mathcal{A}+\Delta_{\mathcal{A}}\right) q(t)+\mathcal{B}\left(\mathcal{F}+\Delta_{\mathcal{F}}\right) q(t)+\mathcal{B}_{w} w(t), \\
z(t)=\mathcal{L} q(t) .
\end{gathered}
$$

According to the assumption (5), Eq. (12) can be written as: 


$$
\begin{gathered}
\dot{q}(t)=\left(\mathcal{E}+\Delta_{\mathcal{E}}\right)^{-1}\left(\mathcal{A}+\Delta_{\mathcal{A}}\right) q(t)+\left(\mathcal{E}+\Delta_{\mathcal{E}}\right)^{-1} \mathcal{B}\left(\mathcal{F}+\Delta_{\mathcal{F}}\right) q(t) \\
+\left(\mathcal{E}+\Delta_{\mathcal{E}}\right)^{-1} \mathcal{B}_{w} w(t)=\left[\overline{\mathcal{A}}+\overline{\mathcal{B}}\left(\mathcal{F}+\Delta_{\mathcal{F}}\right)\right] q(t)+\overline{\mathcal{B}}_{w} w(t),
\end{gathered}
$$

where:

$$
\begin{gathered}
\overline{\mathcal{A}}=\left(\mathcal{E}+\Delta_{\mathcal{E}}\right)^{-1}\left(\mathcal{A}+\Delta_{\mathcal{A}}\right), \\
\overline{\mathcal{B}}=\left(\mathcal{E}+\Delta_{\mathcal{E}}\right)^{-1} \mathcal{B}, \\
\overline{\mathcal{B}}_{w}=\left(\mathcal{E}+\Delta_{\mathcal{E}}\right)^{-1} \mathcal{B}_{w} .
\end{gathered}
$$

According to the Bounded Real Lemma [22-23] for the system (12), following the procedure given in [22] and by applying the Schur complement and by some rearrangement of matrix sub-blocks, the result can be expressed as an LMI. Therefore, for the uncertain system (4) with given $\gamma>0, \eta>0$, a state feedback control of form (9) can be constructed which could tolerate the system uncertainties $\Delta_{\mathrm{M}}, \Delta_{\mathrm{C}}, \Delta_{\mathrm{K}}$, and controller gain variations $\Delta_{\mathrm{F}}$ so that the resulting closed loop system is robustly stable with disturbance attenuation $\gamma$ provided that there exist matrices $\mathbf{X}>0, \mathbf{Q}>0, \mathbf{Y}$ and scalars $\varepsilon>0, \varepsilon_{i}>0$, $i=1,2,3, \mu_{i}>0, i=1,2,3,4$, satisfying the following LMIs:

$$
\begin{aligned}
& {\left[\begin{array}{cccc}
\Omega_{11} & \Omega_{12} & \Omega_{13} & \Omega_{14} \\
* & \Omega_{22} & 0 & 0 \\
* & * & \Omega_{33} & 0 \\
* & * & * & \Omega_{44}
\end{array}\right]<0,} \\
& \Omega_{11}=\eta\left(\mathcal{A} X \mathcal{A}^{T}+\mu_{1} \mathcal{L}_{c} \mathcal{L}_{c}^{T}+\mu_{3} \mathcal{L}_{k} \mathcal{L}_{k}^{T}+\mu_{3} \mathcal{L}_{k} \mathcal{L}_{k}^{T}+\mathcal{A} Q \mathcal{A}^{T}\right) \\
& +\eta^{-1}\left(\mathcal{E X} \mathcal{E}^{T}+\mu_{4} \delta^{2} I\right)+\mathcal{E} Y^{T} \mathcal{B}^{T}+\mathcal{B} Y \mathcal{E}^{T}+\varepsilon_{1} \delta^{2} I+\varepsilon_{2} \delta^{2} I+\varepsilon_{3} \delta^{2} I+\varepsilon \mathcal{B} \mathcal{L}_{f} \mathcal{L}_{f}^{T} \mathcal{B}^{T}, \\
& \Omega_{12}=\left[\begin{array}{lllll}
\mathcal{B}_{w} & \eta \mathcal{A} Q \mathcal{E}_{k}^{T} & \eta \mathcal{A X} \mathcal{E}_{k}^{T} & \mathcal{E} X \mathcal{E}^{T} & \mathcal{B} Y \mathcal{E}^{T}
\end{array}\right], \\
& \Omega_{22}=\operatorname{diag}\left[-\gamma I,-\eta\left(\mu_{3} I-\mathcal{E}_{k} Q \mathcal{E}_{k}^{T}\right),-\eta\left(\mu_{2} I-\mathcal{E}_{k} X \mathcal{E}_{k}^{T}\right),-\eta\left(\mu_{4} I-\mathcal{E} X \mathcal{E}^{T}\right),-\mathcal{E}_{1} I\right], \\
& \Omega_{33}=\left[\begin{array}{cc}
-\gamma I & \mathcal{L} X \mathcal{E}^{T} \\
* & -\varepsilon_{2} I
\end{array}\right], \\
& \Omega_{14}=\left[\begin{array}{llllll}
I & 0 & 0 & 0 & 0 & 0
\end{array}\right]^{T} \mathcal{E} X \mathcal{E}_{f}^{T}\left[\begin{array}{ll}
I & 0
\end{array}\right], \\
& \Omega_{44}=\left[\begin{array}{cc}
-\varepsilon I & \mathcal{E}_{f} X \mathcal{E}^{T} \\
* & -\varepsilon_{3} I
\end{array}\right]
\end{aligned}
$$

and

$$
\left[\begin{array}{cc}
-Q & X \mathcal{E}_{c}^{T} \\
\mathcal{E}_{c} X & -\left(\mu_{1} I-\mathcal{E}_{c} X \mathcal{E}_{c}^{T}\right)
\end{array}\right]<0 .
$$

And, a desired robust non-fragile $H_{\infty}$ state feedback control gain matrix is given by $F=Y X^{1}$. 


\section{NUMERICAL RESULTS AND DISCUSSION}

In order to evaluate the effectiveness and robust performance of the controller design method proposed in the above section, an example is introduced in this section. We assume that the vehicle model is a generic sedan car as the parameters given in Table 1.

Table 1 Half-car model parameters

\begin{tabular}{|c|c|c|c|c|c|c|c|c|c|}
\hline \multicolumn{2}{|c|}{ Mass (kg) } & \multicolumn{2}{|c|}{$\begin{array}{l}\text { Damping } \\
\text { coefficient }(\mathrm{Ns} / \mathrm{m})\end{array}$} & $\begin{array}{l}\text { Spring } \\
(\mathrm{N} / \mathrm{m})\end{array}$ & constant & \multicolumn{2}{|c|}{ Length (m) } & \multicolumn{2}{|c|}{$\begin{array}{l}\text { Moment of Inertia } \\
\left(\mathrm{kg} \cdot \mathrm{m}^{2}\right)\end{array}$} \\
\hline Symbol & Magnitude & Symbol & Magnitude & Symbol & Magnitude & Symbol & Magnitude & Symbol & Magnitude \\
\hline$M_{b}$ & 950 & $C_{s f}$ & 1000 & $K_{t}$ & 200000 & $L_{f}$ & 1.34 & $I_{p}$ & 1500 \\
\hline$M_{t f}$ & 50 & $C_{s r}$ & 1000 & $K_{s f}$ & 39000 & $L_{r}$ & 1.46 & & \\
\hline$M_{t r}$ & 50 & & & $K_{s r}$ & 37000 & $p_{x}$ & 0.04 & & \\
\hline & & & & & & $H_{p}$ & 0.53 & & \\
\hline
\end{tabular}

Table 2 Driver model parameters

\begin{tabular}{lccccr}
\hline Mass (kg) & & \multicolumn{2}{c}{ Damping coefficient (Ns/m) } & \multicolumn{2}{l}{ Spring constant (N/m) } \\
\hline Symbol & Magnitude & Symbol & Magnitude & Symbol & Magnitude \\
\hline$m_{H}$ & 4.17 & $c_{H-U T}$ & 310 & $k_{H-U T}$ & 166990 \\
$m_{U T}$ & 15 & $c_{U T-L T}$ & 200 & $k_{U T-L T}$ & 10000 \\
$m_{L T}$ & 5.5 & $c_{U T-T}$ & 909.1 & $k_{U T-T}$ & 144000 \\
$m_{T}$ & 36 & $c_{L T-T}$ & 330 & $k_{L T-T}$ & 20000 \\
$m_{s e}$ & 35 & $c_{T-s e}$ & 2475 & $k_{T-s e}$ & 49340 \\
& & $c_{s e}$ & 150 & $k_{s e}$ & 15000 \\
\hline
\end{tabular}

As mentioned before, in the biodynamic model the seated human body is constructed with four separate mass segments interconnected by five sets of springs and dampers. With a total human mass of $60.67 \mathrm{~kg}$ the nominal design parameters for the biodynamic model are listed in Table 2. Accordingly, the output variables are chosen to be the displacements and velocities of each mass part, therefore, $L=\mathrm{I}$. The uncertainties in the mass, damping, and stiffness matrices are, respectively, modeled as:

$$
\begin{gathered}
\Delta_{M} M^{-1} \leq \delta=0.1, \\
\Delta_{K}=L_{k} F_{k} E_{k} \equiv(0.1 K) F_{k}(I), \\
\Delta_{c}=L_{c} F_{c} E_{c}=(0.1 C) F_{d}(I) .
\end{gathered}
$$

Assume that the controller gain variation has structure:

$$
\begin{gathered}
\mathfrak{L}_{f}=a_{f}[1,1,1,1,1,1,1,1,1]^{T}, \\
\mathcal{E}_{f}=[1,1,1,1,1,1,1,1,1,1,1,1,1,1,1,1,1,1],
\end{gathered}
$$

where $a_{f}$ is an adjustable parameter to describe the level of gain variation (in the range of $\left.\pm 10^{2}\right)$. 
The frequency responses of the proposed controller are illustrated in Figs. 3 to 6. As it can be seen, the non-fragile controller improves the driver's head acceleration. The designed controller greatly attenuates road excitation, especially in the frequency range around $10 \mathrm{rad} / \mathrm{s}$, the range that affects ride comfort greatly. In addition, Fig. 3 shows that the inaccuracy in the controller gain has no significant effect on the driver's head acceleration in this frequency range. With respect to suspension deflection from Fig. 4, the closed-loop system is not as good as the passive controller, which is an inevitable tradeoff. It is impossible to simultaneously reduce both body acceleration and suspension deflection in the low-frequency range and around the wheel-hop frequency [24]. As for road holding, the proposed controller reduces the value of the first peak in Fig. 5. Moreover, it may result in handling-loss. One has to note that the variation of the controller gain makes no changes in the frequency responses of suspension deflections and road holdings.
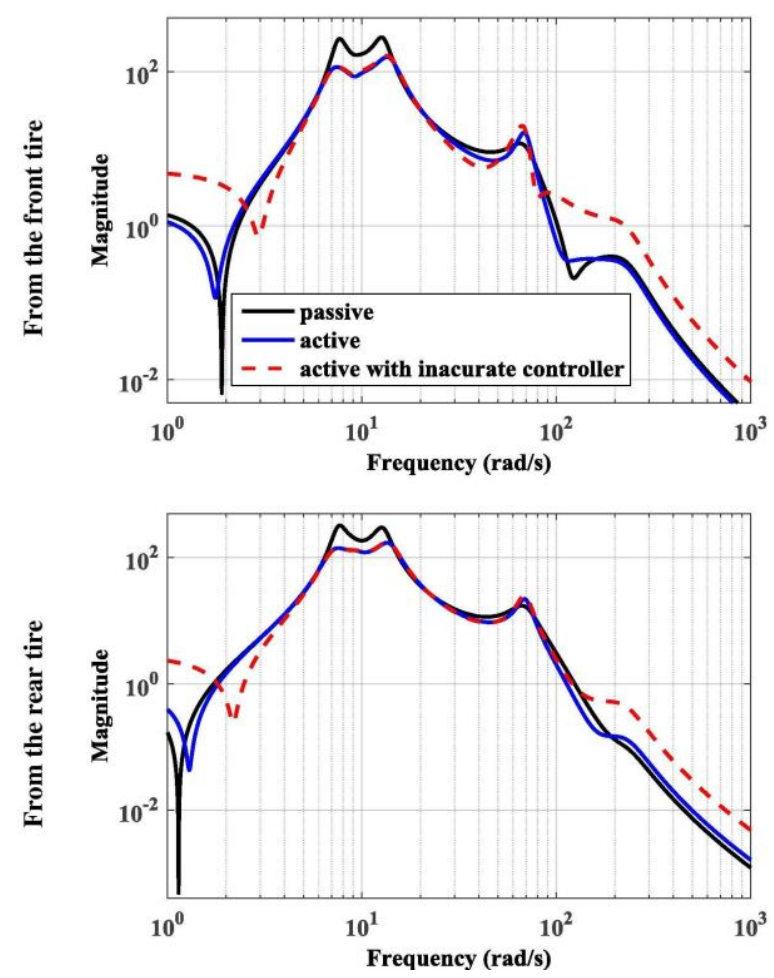

Fig. 3 Frequency response of the head acceleration 

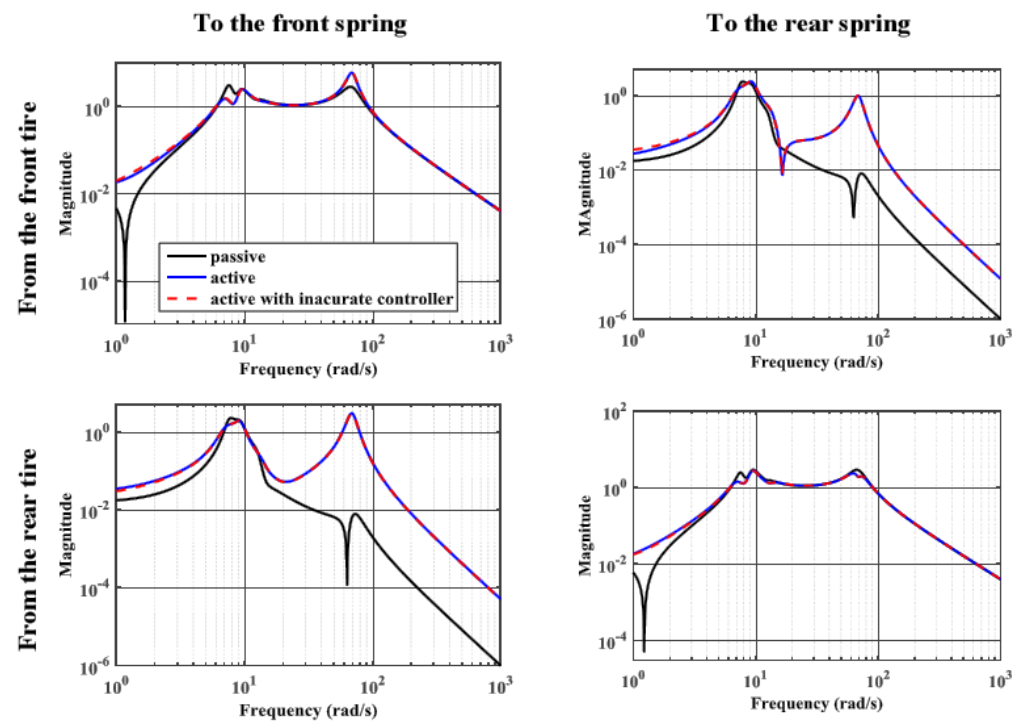

Fig. 4 Frequency response of suspension deflection
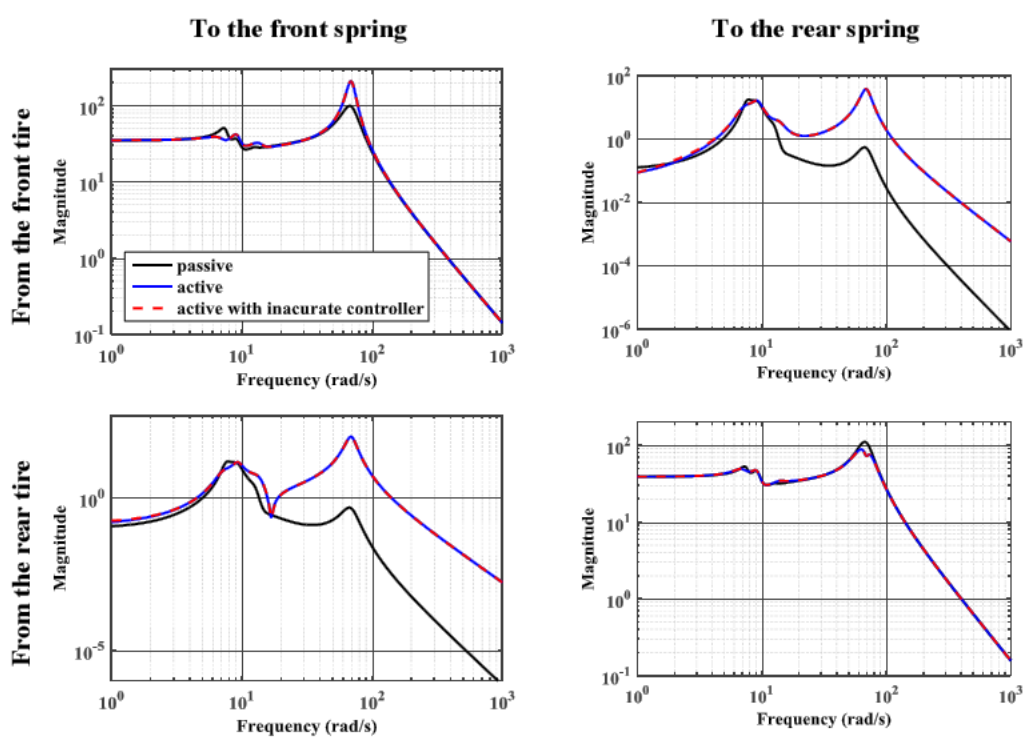

Fig. 5 Frequency response of road holding

The aim of active suspension is to reduce acceleration to the greatest extent, which leads to a good ride comfort, and at the same, to keep the suspension deflection and road holding in acceptable ranges. It means that the performance of the suspension deflection and road holding is sacrificed in order to get a good ride comfort, and therefore the frequency response of this case is what is acceptable. 
The actuator force frequency responses in Fig. 6 are indicators of the energy cost of the two actuators using accurate and inaccurate controllers. This subject is further discussed in the following part of the time responses.
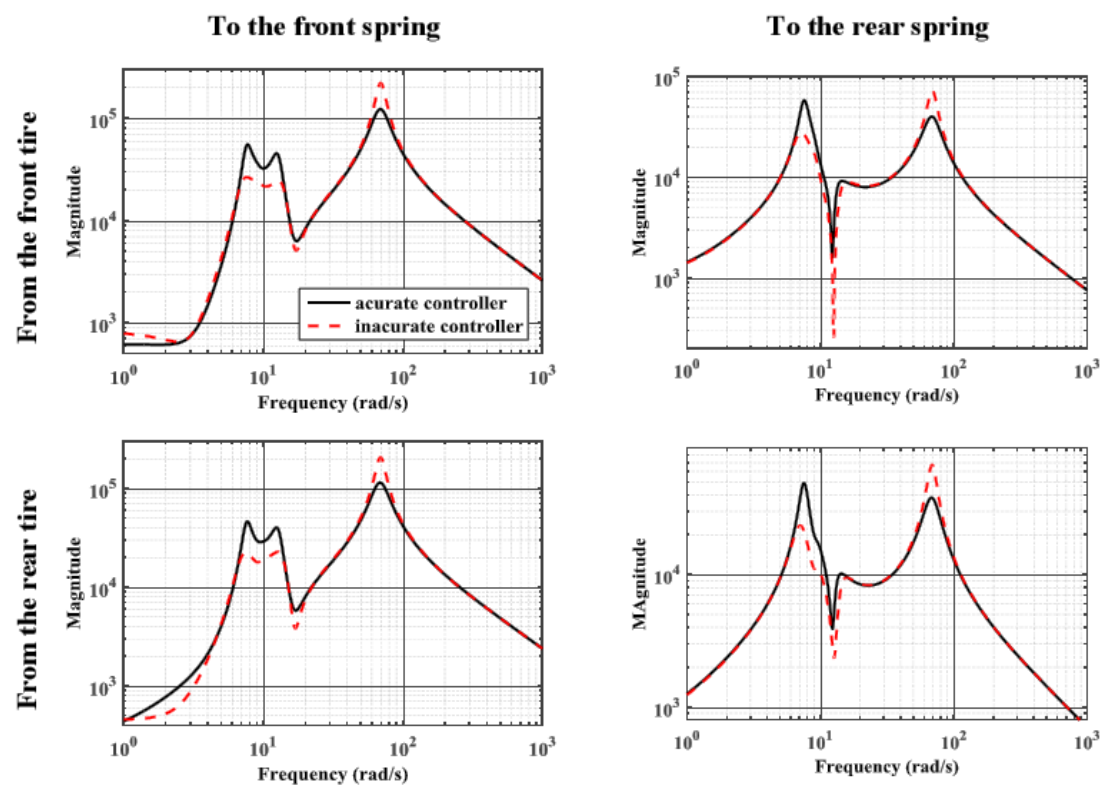

Fig. 6 Frequency response of actuators forces

In order to further test the validity of the designed controller, a set of simulations in the time domain are carried out on a current road excitation. For investigation of the active suspension performance, road disturbances can be generally assumed as shocks which are discrete events of relatively short duration and high intensity, caused by, e.g., a pronounced bump or pothole on an otherwise smooth road surface. In this work, this case of road profile is considered first to reveal the transient response characteristic, which is given by:

$$
z_{0}(t)=\left\{\begin{array}{cc}
\frac{a}{2}\left(1-\cos \left(\frac{2 \pi V_{0}}{l} t\right)\right), & 0 \leq t \leq \frac{l}{V_{0}}, \\
0, & t>\frac{l}{V_{0}},
\end{array}\right.
$$

and illustrated in Fig. 7, where $a$ is the height of the bump, and $l$ is the length of the bump. Here we choose $a=0.1 \mathrm{~m}, l=2 \mathrm{~m}$ and vehicle speed $V_{0}=30 \mathrm{~km} / \mathrm{h}$. 


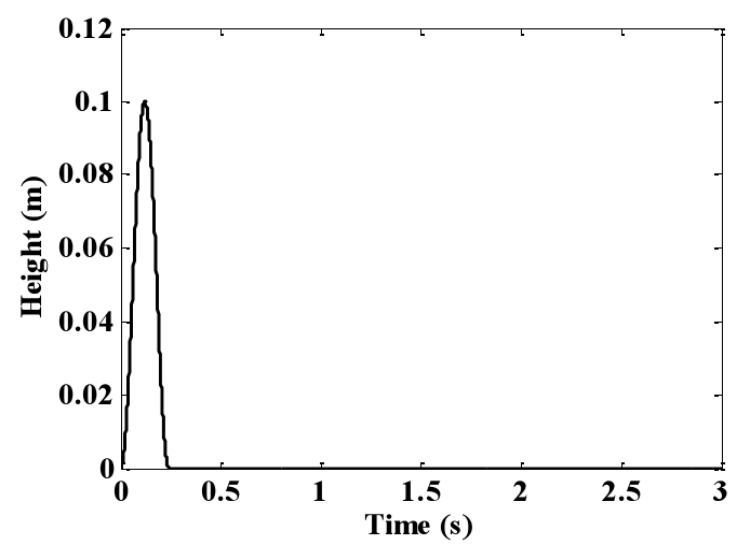

Fig. 7 Bump input

The time responses of the designed controller due to bump disturbance are shown in Figs. 8 to 11. It can be seen that the driver's head acceleration for the designed controller has much lower peaks and the settling time is reduced, as the suspension deflection. The peaks in road holding are a little bigger than for passive suspension. In addition, actuator forces in the time domain are shown in Fig. 11, where produced forces are in an acceptable range which can be generated by hydraulic or electrorheological actuators in practice [2526]. As one can see in these figures, the controller variation does not have a sensible effect on the time responses of the closed-loop system.

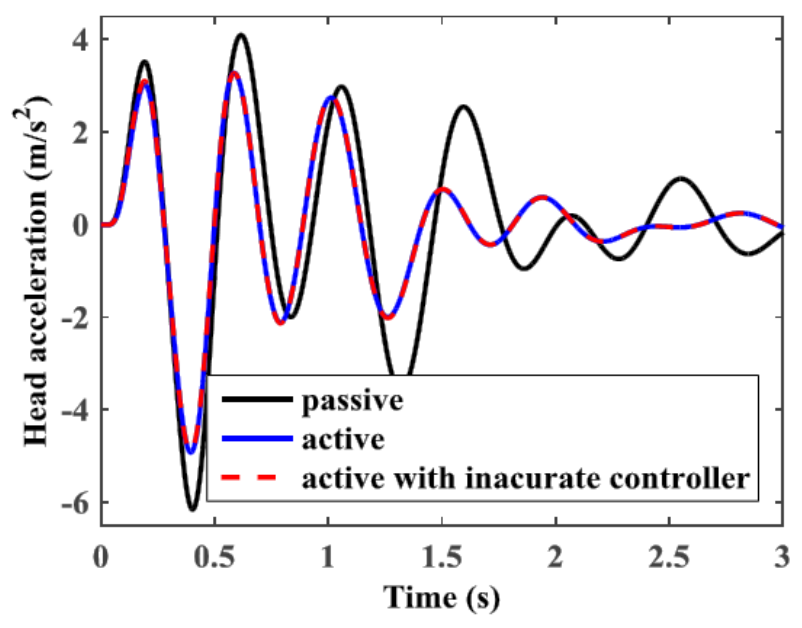

Fig. 8 Head acceleration due to bump excitation 

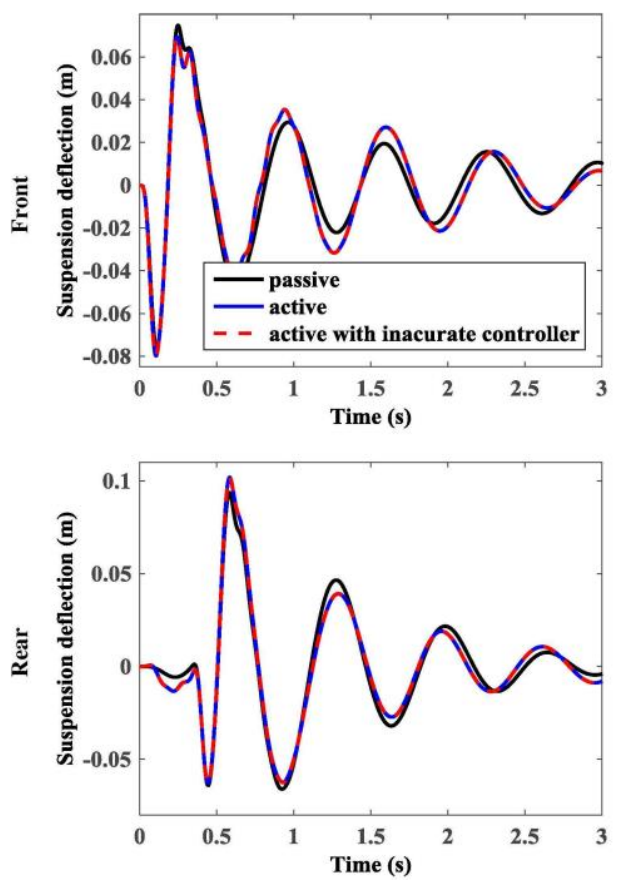

Fig. 9 Suspension deflections due to bump excitation
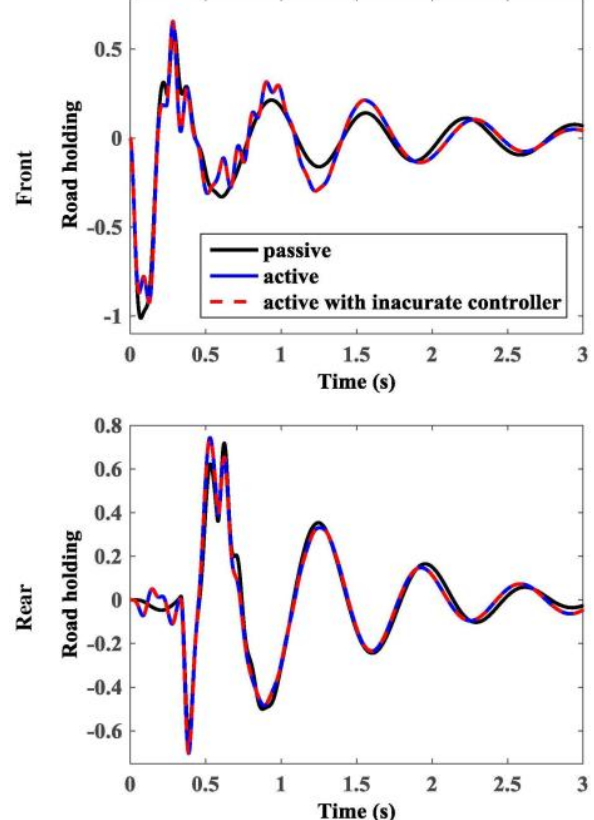

Fig. 10 Road holding due to bump excitation 

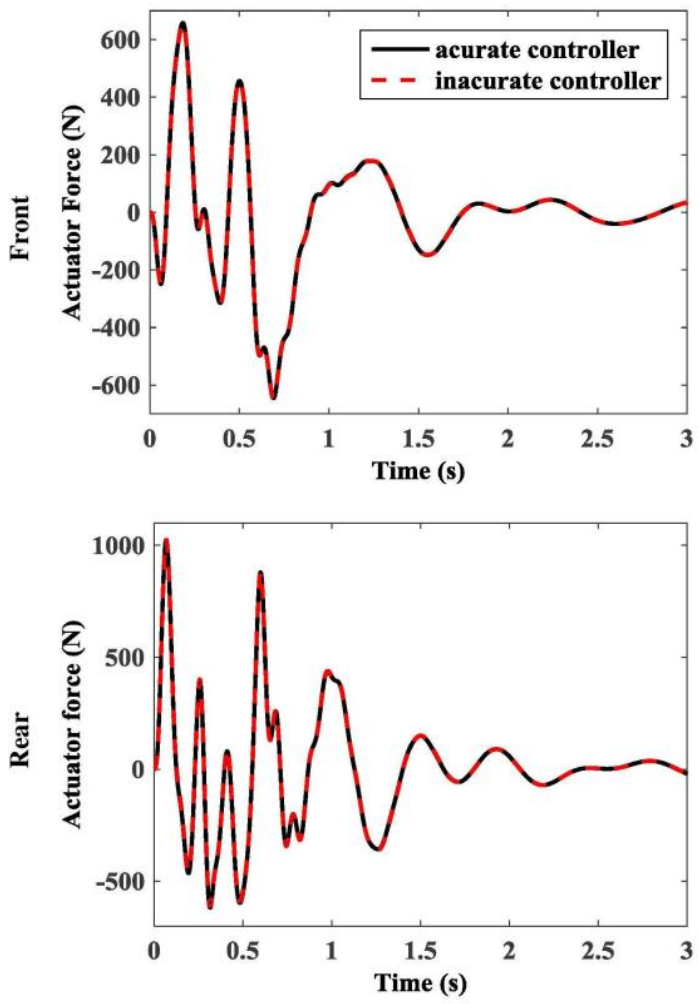

Fig. 11 Actuators forces due to bump excitation

It is confirmed that the designed non-fragile robust controller is able to guarantee a better performance under a pronounced bump disturbance and limited actuator control force in spite of the car-driver's uncertainties and controller variations.

\section{CONCLUSIONS}

Ride performance, suspension deflection, road holding and actuators' forces of a class of half-car suspension systems considering an uncertain 4-DoF driver's biodynamics using a non-fragile robust $H_{\infty}$ state feedback controller is investigated. The biodynamic model has been added to the system to obtain a good tradeoff between performance and accuracy as well as a better insight of the controller design. A design example demonstrates the effectiveness of the proposed controller design approach in comparison with the same passive suspensions and in the presence of the controller gain inaccuracy. Finally, it can be concluded that the proposed controller can successfully deal with the uncertainties in the half-car and driver system and its controller, and it guarantees the ride comfort performance by remaining suspension deflection, road holding and actuators' forces in a reasonable range. 


\section{REFERENCES}

1. El Madany, M.M., Al-Majed, M.I., 2001, Quadratic synthesis of active controls for a quarter-car model, Journal of Vibration and Control, 7(8), pp. 1237-1252.

2. Guo, L.-X., Zhang, L.-P., 2012, Robust Hoo control of active vehicle suspension under non-stationary running, Journal of Sound and Vibration, 331(26), pp. 5824-5837.

3. Gudarzi, M., Oveisi, A., Mohammadi, M.M., 2013, Robust output feedback control for active seat suspension systems with actuator time delay using $\mu$-synthesis approach, Research Journal of Applied Sciences, Engineering and Technology, 6(19), pp. 3559-3567.

4. Li, H., Liu, H., Hand, S., Hilton, C., 2012, Multi-objective Hoo control for vehicle active suspension systems with random actuator delay, International Journal of Systems Science, 43(12), pp. 2214-2227.

5. Eltantawie, M.A., 2012, Decentralized neuro-fuzzy control for half car with semi-active suspension system, International Journal of Automotive Technology, 13(3), pp. 423-431.

6. Gudarzi, M., Oveisi, A., 2015, Ride comfort performance of active vehicle suspension with seat actuator based on non-fragile Ho controller, International Review on Modelling and Simulations (IREMOS), 8(1), pp. 90-98.

7. Mashadi, B., Mahmoudi-Kaleybar, M., Ahmadizadeh, P. and Oveisi, A., 2014, A path-following driver/vehicle model with optimized lateral dynamic controller, Latin American journal of solids and structures, 11(4), pp. 613-630.

8. Tavoosi, V., Kazemi, R. and Oveisi, A., 2014, Nonlinear adaptive optimal control for vehicle handling improvement through steer-by-wire system, Journal of Central South University, 21(1), pp.100-112.

9. ISO 2631-1, 1997, Mechanical vibration and shock-Evaluation of human exposure to whole-body vibrationPart 1: General requirements, International Organization for Standardization.

10. Zhao, Y., Sun, W., Gao, H., 2010, Robust control synthesis for seat suspension systems with actuator saturation and time-varying input delay, Journal of Sound and Vibration, 329(21), pp. 4335-4353.

11. Yamashita, M., Fujimori, K., Hayakawa, K., Kimura, H., 1994, Application of $H \infty 0$ control to active suspension systems, Automatica, 30(11), pp. 1717-1729.

12. Hrovat, D., 1997, Survey of advanced suspension developments and related optimal control applications, Automatica, 33(10), pp. 1781-1817.

13. Ma, M., Chen, H., Liu, X., 2012, Robust H-infinity control for constrained uncertain systems and its application to active suspension, Journal of Control Theory and Applications, 10(4), pp. 470-476.

14. Paattilammi, J., Makila, P.M., 2008, Fragility and robustness: a case study on paper machine headbox control. IEEE Control Systems Magazine, 20(1), pp. 13-22.

15. Norlander, T., Makila, P.M., 2001, Defragilization in optimal design and its application to fixed structure $L Q$ controller design, IEEE Control System Technology, 9(4), pp. 590-598.

16. Dorato, P., 1998, Non-fragile controller design: an overview, Proceedings of the American Control Conference, Philadelphia, pp. 2829-2831.

17. Yee, J.S., Yang, G.H., Wang, J.L., 2000, Non-fragile Ho flight controller design for large bank-angle tracking manoeuvres, Proceedings of the Institution of Mechanical Engineers Part I Journal of Systems and Control Engineering, 214(3), pp. 157-172.

18. Yang, G.H., Wang, J.L., 2001, Non-fragile Hoo control for linear systems with multiplicative controller gain variations, Automatica, 37, pp. 727-737.

19. Yang, G.H., Wang, J.L., Lin, C., 2003, Ho control for linear systems with additive controller gain variations, International Journal of Control, 73(16), pp. 1500-1506.

20. Du, H., Lam, J., Sze, K.Y., 2005, Design of non-fragile Ho controller for active vehicle suspensions. Journal of Vibration and Control, 11(2), pp. 225-243.

21. Gudarzi, M., Oveisi, A., 2014, Robust control for ride comfort improvement of an active suspension system considering uncertain driver's biodynamics, Journal of Low Frequency Noise Vibration and Active Control, 33(3), pp. 317-340.

22. Khargonekar, P.P., Petersen, I.R., Zhou, K., 1990, Robust stabilization of uncertain linear systems: quadratic stabilizability and Hoo control theory, IEEE Transactions on Automatic Control, 35, pp. 356-361.

23. Oveisi, A., Shakeri, R., 2016, Robust reliable control in vibration suppression of sandwich circular plates, Engineering Structures, 116, pp. 1-11.

24. Hedrick, J.K., Butsuen, T., 1990, Invariant properties of automotive suspensions, Proceedings of the Institution of Mechanical Engineers Part D Journal of Automobile Engineering, 204(1), pp. 21-27.

25. Takabi, B., Salehi, S., 2014, Augmentation of the heat transfer performance of a sinusoidal corrugated enclosure by employing hybrid nanofluid, Advances in Mechanical Engineering, 6, doi: 10.1155/2014/147059

26. Gheitaghy, A.M., Takabi, B., Alizadeh, M., 2014, Modeling of ultrashort pulsed laser irradiation in the cornea based on parabolic and hyperbolic heat equations using electrical analogy, International Journal of Modern Physics C, 25(9), doi: 10.1142/S0129183114500399 